\title{
Existence and Multiplicity of Positive Solutions of a Boundary-Value Problem for Sixth-Order ODE with Three Parameters
}

\section{Liyuan Zhang and Yukun An}

Nanjing University of Aeronautics and Astronautics, 29 Yudao st., Nanjing 210016, China

Correspondence should be addressed to Liyuan Zhang, binghaiyiyuan1@163.com

Received 13 May 2010; Accepted 14 August 2010

Academic Editor: Kanishka Perera

Copyright (C) 2010 L. Zhang and Y. An. This is an open access article distributed under the Creative Commons Attribution License, which permits unrestricted use, distribution, and reproduction in any medium, provided the original work is properly cited.

We study the existence and multiplicity of positive solutions of the following boundary-value problem: $-u^{(6)}-\gamma u^{(4)}+\beta u^{\prime \prime}-\alpha u=f(t, u), 0<t<1, u(0)=u(1)=u^{\prime \prime}(0)=u^{\prime \prime}(1)=u^{(4)}(0)=u^{(4)}(1)=$ 0 , where $f:[0,1] \times \mathbf{R}^{+} \rightarrow \mathbf{R}^{+}$is continuous, $\alpha, \beta$, and $\gamma \in \mathbf{R}$ satisfy some suitable assumptions.

\section{Introduction}

The following boundary-value problem:

$$
\begin{aligned}
& u^{(6)}+A u^{(4)}+B u^{\prime \prime}+C u-f(x, u)=0, \quad 0<x<L, \\
& u(0)=u(L)=u^{\prime \prime}(0)=u^{\prime \prime}(L)=u^{(4)}(0)=u^{(4)}(L)=0
\end{aligned}
$$

where $A, B$, and $C$ are some given real constants and $f(x, u)$ is a continuous function on $\mathbf{R}^{2}$, is motivated by the study for stationary solutions of the sixth-order parabolic differential equations

$$
\frac{\partial u}{\partial t}=\frac{\partial^{6} u}{\partial x^{6}}+A \frac{\partial^{4} u}{\partial x^{4}}+B \frac{\partial^{2} u}{\partial x^{2}}+f(x, u)
$$

This equation arose in the formation of the spatial periodic patterns in bistable systems and is also a model for describing the behaviour of phase fronts in materials that are undergoing a 
transition between the liquid and solid state. When $f(x, u)=u-u^{3}$, it was studied by Gardner and Jones [1] as well as by Caginalp and Fife [2].

If $f$ is an even $2 L$-periodic function with respect to $x$ and odd with respect to $u$, in order to get the $2 L$-stationary spatial periodic solutions of (1.2), one turns to study the two points boundary-value problem (1.1). The $2 L$-periodic extension $\bar{u}$ of the odd extension of the solution $u$ of problems (1.1) to the interval $[-L, L]$ yields $2 L$-spatial periodic solutions of (1.2)

Gyulov et al. [3] have studied the existence and multiplicity of nontrivial solutions of BVP (1.1). They gained the following results.

Theorem 1.1. Let $f(x, u): \mathbf{R}^{2} \rightarrow \mathbf{R}$ be a continuous function and $F(x, u)=\int_{0}^{u} f(x, s) d s$. Suppose the following assumptions are held:

$\left(H_{1}\right) F(x, u) / u^{2} \rightarrow+\infty$ as $|u| \rightarrow+\infty$, uniformly with respect to $x$ in bounded intervals,

$\left(H_{2}\right) 0 \leq F(x, u)=o\left(u^{2}\right)$ as $u \rightarrow 0$, uniformly with respect to $x$ in bounded intervals,

then problem (1.1) has at least two nontrivial solutions provided that there exists a natural number $n$ such that $P(n \pi / L)<0$, where $P(\xi)=\xi^{6}-A \xi^{4}+B \xi^{2}-C$ is the symbol of the linear differential operator $L u=u^{(6)}+A u^{(4)}+B u^{\prime \prime}+C u$.

At the same time, in investigating such spatial patterns, some other high-order parabolic differential equations appear, such as the extended Fisher-Kolmogorov (EFK) equation

$$
\frac{\partial u}{\partial t}=-\zeta \frac{\partial^{4} u}{\partial x^{4}}+\frac{\partial^{2} u}{\partial x^{2}}+u-u^{3}, \quad \zeta>0,
$$

proposed by Coullet, Elphick, and Repaux in 1987 as well as by Dee and Van Saarlos in 1988 and Swift-Hohenberg (SH) equation

$$
\frac{\partial u}{\partial t}=\rho u-\left(1+\frac{\partial^{2} u}{\partial x^{2}}\right)^{2} u-u^{3}, \quad \rho>0,
$$

proposed in 1977.

In much the same way, the existence of spatial periodic solutions of both the EFK equation and the SH equation was studied by Peletier and Troy [4], Peletier and Rottschäfer [5], Tersian and Chaparova [6], and other authors. More precisely, in those papers, the authors studied the following fourth-order boundary-value problem:

$$
\begin{gathered}
u^{(4)}+A u^{\prime \prime}+B u+f(x, u)=0, \quad 0<x<L, \\
u(0)=u(L)=u^{\prime \prime}(0)=u^{\prime \prime}(L)=0 .
\end{gathered}
$$

The methods used in those papers are variational method and linking theorems.

On the other hand, The positive solutions of fourth-order boundary value problems (1.5) have been studied extensively by using the fixed point theorem of cone extension or compression. Here, we mention Li's paper [7], in which the author decomposes the fourth-order differential operator into the product of two second-order differential operators 
to obtain Green's function and then used the fixed point theorem of cone extension or compression to study the problem.

The purpose of this paper is using the idea of [7] to investigate BVP for sixthorder equations. We will discuss the existence and multiplicity of positive solutions of the boundary-value problem

$$
\begin{gathered}
-u^{(6)}-\gamma u^{(4)}+\beta u^{\prime \prime}-\alpha u=f(t, u), \quad 0<t<1, \\
u(0)=u(1)=u^{\prime \prime}(0)=u^{\prime \prime}(1)=u^{(4)}(0)=u^{(4)}(1)=0,
\end{gathered}
$$

and then we assume the following conditions throughout:

(H1) $f:[0,1] \times[0, \infty) \mapsto[0, \infty)$ is continuous,

(H2) $\alpha, \beta$, and $\gamma \in \mathbf{R}$ satisfy

$$
\begin{gathered}
\gamma<3 \pi^{2}, \quad 3 \pi^{4}-2 \gamma \pi^{2}-\beta>0, \\
\frac{\alpha}{\pi^{6}}+\frac{\beta}{\pi^{4}}+\frac{\gamma}{\pi^{2}}<1, \\
18 \alpha \beta \gamma-\beta^{2} \gamma^{2}+4 \alpha \gamma^{3}+27 \alpha^{2}-4 \beta^{3} \leq 0 .
\end{gathered}
$$

Note. The set of $\alpha, \beta$, and $\gamma$ which satisfies (H2) is nonempty. For instance, if $\gamma=\pi^{2}, \beta=0$, then (H2) holds for $\alpha$ : $-4 \pi^{2} / 27<\alpha<0$.

To be convenient, we introduce the following notations:

$$
\begin{gathered}
L=\pi^{6}-\gamma \pi^{4}-\beta \pi^{2}-\alpha, \\
\underline{f}_{0}=\liminf _{u \rightarrow 0+} \min _{t \in[0,1]}\left(\frac{f(t, u)}{u}\right), \quad \bar{f}_{\infty}=\limsup _{u \rightarrow \infty} \max _{t \in[0,1]}\left(\frac{f(t, u)}{u}\right), \\
\underline{f}_{-\infty}=\liminf _{u \rightarrow \infty} \min _{t \in[0,1]}\left(\frac{f(t, u)}{u}\right), \quad \bar{f}_{0}=\limsup _{u \rightarrow 0+} \max _{t \in[0,1]}\left(\frac{f(t, u)}{u}\right) .
\end{gathered}
$$

\section{Preliminaries}

Lemma 2.1 (see [8]). Set the cubic equation with one variable as follows:

$$
a x^{3}+b x^{2}+c x+d=0, \quad a, b, c, d \in \mathbf{R}, a \neq 0 .
$$

Let

$$
A=b^{2}-3 a c, \quad B=b c-9 a d, \quad C=c^{2}-3 b d, \quad \Delta=B^{2}-4 A C,
$$

one has the following:

(1) Equation (2.1) has a triple root if $A=B=0$,

(2) Equation (2.1) has a real root and two mutually conjugate imaginary roots if $\Delta=B^{2}$ $4 A C>0$, 
(3) Equation (2.1) has three real roots, two of which are reroots if $\Delta=B^{2}-4 A C=0$,

(4) Equation (2.1) has three unequal real roots if $\Delta=B^{2}-4 A C<0$.

Lemma 2.2. Let $\lambda_{1}, \lambda_{2}$, and $\lambda_{3}$ be the roots of the polynomial $P(\lambda)=\lambda^{3}+\gamma \lambda^{2}-\beta \lambda+\alpha$. Suppose that condition (H2) holds, then $\lambda_{1}, \lambda_{2}$, and $\lambda_{3}$ are real and greater than $-\pi^{2}$.

Proof. There are $A=\gamma^{2}+3 \beta, B=-\beta \gamma-9 \alpha$, and $C=\beta^{2}-3 \alpha \gamma$ in the equation $P(\lambda)=0$. Since condition (H2) holds, we have

$$
\Delta=B^{2}-4 A C=18 \alpha \beta \gamma-\beta^{2} \gamma^{2}+4 \alpha \gamma^{3}+27 \alpha^{2}-4 \beta^{3} \leq 0
$$

Therefore, the equation has three real roots in reply to Lemma 2.1.

By Vieta theorem, we have

$$
\begin{gathered}
\lambda_{1} \lambda_{2} \lambda_{3}=-\alpha, \\
\lambda_{1}+\lambda_{2}+\lambda_{3}=-\gamma, \\
\lambda_{1} \lambda_{2}+\lambda_{1} \lambda_{3}+\lambda_{2} \lambda_{3}=-\beta .
\end{gathered}
$$

Therefore $\alpha / \pi^{6}+\beta / \pi^{4}+\gamma / \pi^{2}<1, \gamma<3 \pi^{2}$ and $3 \pi^{4}-2 \gamma \pi^{2}-\beta>0$ hold if and only if

$$
\begin{gathered}
\left(\lambda_{1}+\pi^{2}\right)\left(\lambda_{2}+\pi^{2}\right)\left(\lambda_{3}+\pi^{2}\right)>0 \\
\left(\lambda_{1}+\pi^{2}\right)+\left(\lambda_{2}+\pi^{2}\right)+\left(\lambda_{3}+\pi^{2}\right)>0 \\
\left(\lambda_{1}+\pi^{2}\right)\left(\lambda_{2}+\pi^{2}\right)+\left(\lambda_{1}+\pi^{2}\right)\left(\lambda_{3}+\pi^{2}\right)+\left(\lambda_{2}+\pi^{2}\right)\left(\lambda_{3}+\pi^{2}\right)>0 .
\end{gathered}
$$

Then, we only prove that the system of inequalities (2.5) holds if and only if $\lambda_{1}, \lambda_{2}$, and $\lambda_{3}$ are all greater than $-\pi^{2}$.

In fact, the sufficiency is obvious, we just prove the necessity. Assume that $\lambda_{1}, \lambda_{2}, \lambda_{3}$ are less than $-\pi^{2}$. By the first inequality of (2.5), there exist two roots which are less than $-\pi^{2}$ and one which is greater than $-\pi^{2}$. Without loss of generality, we assume that $\lambda_{2}<-\pi^{2}, \lambda_{3}<$ $-\pi^{2}$, then we have $\lambda_{1}>-\pi^{2}$. Multiplying the second inequality of (2.5) by $\lambda_{2}+\pi^{2}$, one gets

$$
\left(\lambda_{1}+\pi^{2}\right)\left(\lambda_{2}+\pi^{2}\right)+\left(\lambda_{2}+\pi^{2}\right)^{2}+\left(\lambda_{2}+\pi^{2}\right)\left(\lambda_{3}+\pi^{2}\right)<0
$$

Compare with the third inequality of (2.5), we have

$$
\left(\lambda_{2}+\pi^{2}\right)^{2}<\left(\lambda_{1}+\pi^{2}\right)\left(\lambda_{3}+\pi^{2}\right)<0,
$$

which is a contradiction. Hence, the assumption is false. The proof is completed. 
Let $G_{i}(t, s)(i=1,2,3)$ be Green's function of the linear boundary-value problem

$$
-u^{\prime \prime}(t)+\lambda_{i} u(t)=0, \quad u(0)=u(1)=0
$$

Lemma 2.3 (see [7]). $G_{i}(t, s)(i=1,2,3)$ has the following properties:

(i) $G_{i}(t, s)>0$, for all $t, s \in(0,1)$,

(ii) $G_{i}(t, s) \leq C_{i} G_{i}(s, s)$, for all $t, s \in[0,1]$, where $C_{i}>0$ is a constant,

(iii) $G_{i}(t, s) \geq \delta_{i} G_{i}(t, t) G_{i}(s, s)$, for all $t, s \in[0,1]$, where $\delta_{i}>0$ is a constant.

One denotes the following:

$$
\begin{gathered}
M_{i}=\max _{0 \leq s \leq 1} G_{i}(s, s), \quad m_{i}=\min _{1 / 4 \leq s \leq 3 / 4} G_{i}(s, s) \quad(i=1,2,3), \\
C_{12}=\int_{0}^{1} G_{1}(\delta, \delta) G_{2}(\delta, \delta) d \delta, \quad C_{23}=\int_{0}^{1} G_{2}(s, s) G_{3}(s, s) d s,
\end{gathered}
$$

then $M_{i}, m_{i}, C_{12}, C_{23}>0$. Let $\|\cdot\|$ be the maximum norm of $C[0,1]$, and let $C^{+}[0,1]$ be the cone of all nonnegative functions in $C[0,1]$.

Let $h \in C[0,1]$, then one considers linear boundary-value problem (LBVP) as follows:

$$
-u^{(6)}-\gamma u^{(4)}+\beta u^{\prime \prime}-\alpha u=h(t), \quad t \in[0,1],
$$

with the boundary condition (1.7). Since

$$
-u^{(6)}-\gamma u^{(4)}+\beta u^{\prime \prime}-\alpha u=\left(-\frac{d^{2}}{d t^{2}}+\lambda_{1}\right)\left(-\frac{d^{2}}{d t^{2}}+\lambda_{2}\right)\left(-\frac{d^{2}}{d t^{2}}+\lambda_{3}\right) u,
$$

the solution of LBVP (2.10)-(1.7) can be expressed by

$$
u(t)=\iiint_{0}^{1} G_{1}(t, \delta) G_{2}(\delta, \tau) G_{3}(\tau, s) h(s) d s d \tau d \delta
$$

Lemma 2.4. Let $h \in C^{+}[0,1]$, then the solution of $\operatorname{LBVP}(2.10)-(1.7)$ satisfies

$$
u(t) \geq \frac{\delta_{1} \delta_{2} \delta_{3} C_{12} C_{23}}{C_{1} C_{2} C_{3} M_{1} M_{2}} G_{1}(t, t)\|u\|
$$

Proof. From (2.12) and (ii) of Lemma 2.3, it is easy to see that

$$
u(t) \leq C_{1} C_{2} C_{3} M_{1} M_{2} \int_{0}^{1} G_{3}(s, s) h(s) d s,
$$


and, therefore,

$$
\|u\| \leq C_{1} C_{2} C_{3} M_{1} M_{2} \int_{0}^{1} G_{3}(s, s) h(s) d s,
$$

that is,

$$
\int_{0}^{1} G_{3}(s, s) h(s) d s \geq \frac{\|u\|}{C_{1} C_{2} C_{3} M_{1} M_{2}}
$$

Using (iii) of Lemma 2.3, we have

$$
\begin{aligned}
u(t) & =\iiint_{0}^{1} G_{1}(t, \delta) G_{2}(\delta, \tau) G_{3}(\tau, s) h(s) d s d \tau d \delta \\
& \geq \delta_{1} \delta_{2} \delta_{3} C_{12} C_{23} G_{1}(t, t) \int_{0}^{1} G_{3}(s, s) h(s) d s \\
& \geq \frac{\delta_{1} \delta_{2} \delta_{3} C_{12} C_{23} G_{1}(t, t)}{C_{1} C_{2} C_{3} M_{1} M_{2}}\|u\| .
\end{aligned}
$$

The proof is completed.

We now define a mapping $A: C[0,1]^{+} \rightarrow C[0,1]^{+}$by

$$
A u(t)=\iiint_{0}^{1} G_{1}(t, \delta) G_{2}(\delta, \tau) G_{3}(\tau, s) f(s, u(s)) d s d \tau d \delta
$$

It is clear that $A: C[0,1]^{+} \rightarrow C[0,1]^{+}$is completely continuous. By Lemma 2.4 , the positive solution of $\operatorname{BVP}(1.6)-(1.7)$ is equivalent to nontrivial fixed point of $A$. We will find the nonzero fixed point of $A$ by using the fixed point index theory in cones. For this, one chooses the subcone $K$ of $C[0,1]^{+}$by

$$
K=\left\{u \in C[0,1]^{+} \mid u(t) \geq \sigma\|u\|, \forall t \in\left[\frac{1}{4}, \frac{3}{4}\right]\right\}
$$

where $\sigma=\delta_{1} \delta_{2} \delta_{3} C_{12} C_{23} m_{1} / C_{1} C_{2} C_{3} M_{1} M_{2}$, we have the following.

Lemma 2.5. Having $A(K) \subseteq K, A: K \rightarrow K$ is completely continuous.

Proof. For $u \in K$, let $h(t)=f(t, u(t))$, then $A u(t)$ is the solution of $\operatorname{LBVP}(2.10)-(1.7)$. By Lemma 2.4, one has

$$
A u(t) \geq \frac{\delta_{1} \delta_{2} \delta_{3} C_{12} C_{23}}{C_{1} C_{2} C_{3} M_{1} M_{2}} G_{1}(t, t)\|A(u)\| \geq \sigma\|A(u)\|, \quad \forall t \in\left[\frac{1}{4}, \frac{3}{4}\right]
$$

namely $A u \in K$. Therefore, $A(K) \subseteq K$. The complete continuity of $A$ is obvious. 
The main results of this paper are based on the theory of fixed point index in cones [9]. Let $E$ be a Banach space and $K \subset E$ be a closed convex cone in $E$. Assume that $\Omega$ is a bounded open subset of $E$ with boundary $\partial \Omega$, and $K \cap \Omega \neq \emptyset$. Let $A: K \cap \bar{\Omega} \rightarrow K$ be a completely continuous mapping. If $A u \neq u$ for every $u \in K \cap \partial \Omega$, then the fixed point index $i(A, K \cap \Omega, K)$ is well defined. We have that if $i(A, K \cap \Omega, K) \neq 0$, then $A$ has a fixed point in $K \cap \partial \Omega$.

Let $K_{r}=\{u \in K \mid\|u\|<r\}$ and $\partial K_{r}=\{u \in K \mid\|u\|=r\}$ for every $r>0$.

Lemma 2.6 (see [9]). Let $A: K \rightarrow K$ be a completely continuous mapping. If $\mu A u \neq u$ for every $u \in \partial K_{r}$ and $0<\mu \leq 1$, then $i\left(A, K_{r}, K\right)=1$.

Lemma 2.7 (see [9]). Let $A: K \rightarrow K$ be a completely continuous mapping. Suppose that the following two conditions are satisfied:

(i) $\inf _{u \in \partial K_{r}}\|A(u)\|>0$,

(ii) $\mu A u \neq u$ for every $u \in \partial K_{r}$ and $\mu \geq 1$,

then $i\left(A, K_{r}, K\right)=0$.

Lemma 2.8 (see [9]). Let $X$ be a Banach space, and let $K \subseteq X$ be a cone in $X$. For $p>0$, define $K_{p}=\{u \in K \mid\|u\|<p\}$. Assume that $A: K_{p} \rightarrow K$ is a completely continuous mapping such that Au $\neq u$ for every $u \in \partial K_{p}=\{u \in K \mid\|u\|=p\}$.

(i) If $\|u\| \leq\|A u\|$ for every $u \in \partial K_{p}$, then $i\left(A, K_{p}, K\right)=0$.

(ii) If $\|u\| \geq\|A u\|$ for every $u \in \partial K_{p}$, then $i\left(A, K_{p}, K\right)=1$.

\section{Existence}

We are now going to state our existence results.

Theorem 3.1. Assume that (H1) and (H2) hold, then in each of the following case:

(i) $\bar{f}_{0}<L, \underline{f}_{\infty}>L$,

(ii) $\underline{f}_{0}>L, \bar{f}_{\infty}<L$,

the BVP(1.6)-(1.7) has at least one positive solution.

Proof. To prove Theorem 3.1, we just show that the mapping $A$ defined by (2.18) has a nonzero fixed point in the cases, respectively.

Case(i): since $\bar{f}_{0}<L$, by the definition of $\bar{f}_{0}$, we may choose $\varepsilon>0$ and $\omega>0$, so that

$$
f(t, u) \leq(L-\varepsilon) u, \quad 0 \leq t \leq 1,0 \leq u \leq \omega .
$$

Let $r \in(0, \omega)$, we now prove that $\mu A u \neq u$ for every $u \in \partial K_{r}$ and $0<\mu \leq 1$. In fact, if there exist $u_{0} \in \partial K_{r}$ and $0<\mu_{0} \leq 1$ such that $\mu_{0} A u_{0}=u_{0}$, then, by definition of $A, u_{0}(t)$ satisfies differential equation the following:

$$
-u_{0}^{(6)}-\gamma u_{0}^{(4)}+\beta u_{0}^{\prime \prime}-\alpha u_{0}=\mu_{0} f\left(t, u_{0}\right), \quad 0 \leq t \leq 1,
$$


and boundary condition (1.7). Multiplying (3.2) by $\sin \pi t$ and integrating on [0,1], then using integration by parts in the left side, we have

$$
L \int_{0}^{1} u_{0}(t) \sin \pi t d t=\mu_{0} \int_{0}^{1} f\left(t, u_{0}(t)\right) \sin \pi t d t \leq(L-\varepsilon) \int_{0}^{1} u_{0}(t) \sin \pi t d t
$$

By Lemma 2.4, $u(t) \geq\left(\delta_{1} \delta_{2} \delta_{3} C_{12} C_{23} / C_{1} C_{2} C_{3} M_{1} M_{2}\right) G_{1}(t, t)\|u\|$, and then $\int_{0}^{1} u_{0}(t) \sin \pi t d t>$ 0 . We see that $L \leq(L-\varepsilon)$, which is a contradiction. Hence, $A$ satisfies the hypotheses of Lemma 2.6, in $K_{r}$. By Lemma 2.6 we have

$$
i\left(A, K_{r}, K\right)=1 \text {. }
$$

On the other hand, since $\underline{f}_{-\infty}>L$, there exist $\varepsilon \in(0, L)$ and $H>0$ such that

$$
f(t, u) \geq(L+\varepsilon) u, \quad 0 \leq t \leq 1, u \geq H
$$

Let $C=\max _{0 \leq t \leq 1,0 \leq u \leq H}|f(t, u)-(L+\varepsilon) u|+1$, then it is clear that

$$
f(t, u) \geq(L+\varepsilon) u-C, \quad 0 \leq t \leq 1, u \geq 0 .
$$

Choose $R>R_{0}=\max \{H / \sigma, \omega\}$. Let $u \in \partial K_{R}$. Since $u(s) \geq \sigma\|u\|>H$, for all $s \in[1 / 4,3 / 4]$, from (3.5) we see that

$$
f(t, u) \geq(L+\varepsilon) u(s) \geq(L+\varepsilon) \sigma\|u\|, \quad \forall s \in\left[\frac{1}{4}, \frac{3}{4}\right]
$$

By Lemma 2.5, we have

$$
\begin{aligned}
A u\left(\frac{1}{2}\right) & =\iiint_{0}^{1} G_{1}\left(\frac{1}{2}, \delta\right) G_{2}(\delta, \tau) G_{3}(\tau, s) f(s, u(s)) d s d \tau d \delta \\
& \geq \delta_{1} \delta_{2} \delta_{3} C_{12} C_{23} m_{1} \int_{1 / 4}^{3 / 4} G_{3}(s, s) f(s, u(s)) d s \\
& \geq \frac{1}{2} \delta_{1} \delta_{2} \delta_{3} C_{12} C_{23} m_{1} m_{3}(L+\varepsilon) \sigma\|u\| .
\end{aligned}
$$

Therefore,

$$
\|A u\| \geq A u\left(\frac{1}{2}\right) \geq \frac{1}{2} \delta_{1} \delta_{2} \delta_{3} C_{12} C_{23} m_{1} m_{3}(L+\varepsilon) \sigma\|u\|,
$$


from which we see that $\inf _{u \in \partial K_{R}}\|A(u)\|>0$, namely the hypotheses (i) of Lemma 2.7 holds. Next, we show that if $R$ is large enough, then $\mu A u \neq u$ for any $u \in \partial K_{R}$ and $\mu \geq 1$. In fact, if there exist $u_{0} \in \partial K_{R}$ and $\mu_{0} \geq 1$ such that $\mu_{0} A u_{0}=u_{0}$, then $u_{0}(t)$ satisfies (3.2) and boundary condition (1.7). Multiplying (3.2) by $\sin \pi t$ and integrating, from (3.6) we have

$$
L \int_{0}^{1} u_{0}(t) \sin \pi t d t=\mu_{0} \int_{0}^{1} f\left(t, u_{0}(t)\right) \sin \pi t d t \geq(L+\varepsilon) \int_{0}^{1} u_{0}(t) \sin \pi t d t-\frac{2 C}{\pi} .
$$

Consequently, we obtain that

$$
\int_{0}^{1} u_{0}(t) \sin \pi t d t \leq \frac{2 C}{\pi \varepsilon}
$$

By Lemma 2.4,

$$
\int_{0}^{1} u_{0}(t) \sin \pi t d t \geq \frac{\delta_{1} \delta_{2} \delta_{3} C_{12} C_{23}}{C_{1} C_{2} C_{3} M_{1} M_{2}}\left\|u_{0}\right\| \int_{0}^{1} G_{1}(t, t) \sin \pi t d t
$$

from which and from (3.11) we get that

$$
\left\|u_{0}\right\| \leq \frac{2 C C_{1} C_{2} C_{3} M_{1} M_{2}}{\delta_{1} \delta_{2} \delta_{3} C_{12} C_{23} \pi \varepsilon}\left(\int_{0}^{1} G_{1}(t, t) \sin \pi t d t\right)^{-1}:=\bar{R} .
$$

Let $R>\max \left\{\bar{R}, R_{0}\right\}$, then for any $u \in \partial K_{R}$ and $\mu \geq 1, \mu A u \neq u$. Hence, hypothesis (ii) of Lemma 2.7 also holds. By Lemma 2.7, we have

$$
i\left(A, K_{R}, K\right)=0 .
$$

Now, by the additivity of fixed point index, combine (3.4) and (3.14) to conclude that

$$
i\left(A, K_{R} \backslash \overline{K_{r}}, K\right)=i\left(A, K_{R}, K\right)-i\left(A, K_{r}, K\right)=-1
$$

Therefore, $A$ has a fixed point in $K_{R} \backslash \overline{K_{r}}$, which is the positive solution of BVP(1.6)-(1.7).

Case (ii): since $\underline{f}_{0}>L$, there exist $\varepsilon>0$ and $r_{0}>0$ such that

$$
f(t, u) \geq(L+\varepsilon) u, \quad 0 \leq t \leq 1,0 \leq u \leq r_{0} .
$$

Let $r \in\left(0, r_{0}\right)$, then for every $u \in \partial K_{r}$, through the argument used in (3.9), we have

$$
\|A u\| \geq A u\left(\frac{1}{2}\right) \geq \frac{1}{2} \delta_{1} \delta_{2} \delta_{3} C_{12} C_{23} m_{1} m_{3}(L+\varepsilon) \sigma\|u\| .
$$


Hence, $\inf _{u \in \partial K_{r}}\|A(u)\|>0$. Next, we show that $\mu A u \neq u$ for any $u \in \partial K_{r}$ and $\mu \geq 1$. In fact, if there exist $u_{0} \in \partial K_{r}$ and $\mu_{0} \geq 1$ such that $\mu_{0} A u_{0}=u_{0}$, then $u_{0}(t)$ satisfies (3.2) and boundary (1.7). From (3.2) and (3.16), it follows that

$$
L \int_{0}^{1} u_{0}(t) \sin \pi t d t=\mu_{0} \int_{0}^{1} f\left(t, u_{0}(t)\right) \sin \pi t d t \geq(L+\varepsilon) \int_{0}^{1} u_{0}(t) \sin \pi t d t
$$

Since $\int_{0}^{1} u_{0}(t) \sin \pi t d t>0$, we see that $L \geq(L+\varepsilon)$, which is a contradiction. Hence, by Lemma 2.7, we have

$$
i\left(A, K_{r}, K\right)=0 .
$$

On the other hand, since $\bar{f}_{\infty}<L$, there exist $\varepsilon \in(0, L)$ and $H>0$ such that

$$
f(t, u) \leq(L-\varepsilon) u, \quad 0 \leq t \leq 1, u \geq H
$$

Set $C=\max _{0 \leq t \leq 1,0 \leq u \leq H}|f(t, u)-(L-\varepsilon) u|+1$, we obviously have

$$
f(t, u) \leq(L-\varepsilon) u+C, \quad 0 \leq t \leq 1, u \geq 0 .
$$

If there exist $u_{0} \in K$ and $0<\mu_{0} \leq 1$ such that $\mu_{0} A u_{0}=u_{0}$, then (3.2) is valid. From (3.2) and (3.21), it follows that

$$
L \int_{0}^{1} u_{0}(t) \sin \pi t d t=\mu_{0} \int_{0}^{1} f\left(t, u_{0}(t)\right) \sin \pi t d t \leq(L-\varepsilon) \int_{0}^{1} u_{0}(t) \sin \pi t d t+\frac{2 C}{\pi} .
$$

By the proof of (3.13), we see that $\left\|u_{0}\right\| \leq \bar{R}$. Let $R>\max \left\{\bar{R}, r_{0}\right\}$, then for any $u \in \partial K_{R}$ and $0<\mu \leq 1, \mu A u \neq u$. Therefore, by Lemma 2.6, we have

$$
i\left(A, K_{R}, K\right)=1 .
$$

From (3.19) and (3.23), it follows that

$$
i\left(A, K_{R} \backslash \overline{K_{r}}, K\right)=i\left(A, K_{R}, K\right)-i\left(A, K_{r}, K\right)=1 .
$$

Therefore, $A$ has a fixed point in $K_{R} \backslash \overline{K_{r}}$, which is the positive solution of BVP(1.6)-(1.7). The proof is completed. 
From Theorem 3.1, we immediately obtain the following.

Corollary 3.2. Assume that (H1) and (H2) hold, then in each of the following cases:

(i) $\bar{f}_{0}=0, \underline{f}=\infty$,

(ii) $\bar{f}_{\infty}=0, \underline{f}_{0}=\infty$,

the BVP(1.6)-(1.7) has at least one positive solution.

\section{Multiplicity}

Next, we study the multiplicity of positive solutions of BVP(1.6)-(1.7) and assume in this section that

(H3) there is a $p>0$ such that $0 \leq u \leq p$ and $0 \leq t \leq 1$ imply $f(t, u)<\eta p$, where $\eta=\left(C_{1} C_{2} C_{3} M_{1} M_{2} \int_{0}^{1} G_{3}(s, s) d s\right)^{-1}$.

(H4) there is a $p>0$ such that $\sigma p \leq u \leq p$ and $0 \leq t \leq 1$ imply $f(t, u) \geq v p$, where $v^{-1}=\delta_{1} \delta_{2} \delta_{3} C_{12} C_{23} m_{1} \int_{1 / 4}^{3 / 4} G_{3}(s, s) d s$.

Theorem 4.1. If $\underline{f}_{0}>L$ and $\underline{f}_{\infty}>L$ and (H3) is satisfied, then BVP(1.6)-(1.7) has at least two positive solutions: $u_{1}$ and $u_{2}$, such that $0 \leq\left\|u_{1}\right\| \leq p \leq\left\|u_{2}\right\|$.

Proof. According to the proof of Theorem 3.1, there exists $0<r_{0}<p<R_{1}<+\infty$, such that $0<r<r_{0}$ implies $i\left(A, K_{r}, K\right)=0$ and $R \geq R_{1}$ implies $i\left(A, K_{R}, K\right)=0$.

We now prove that $i\left(A, K_{p}, K\right)=1$ if $(H 3)$ is satisfied. In fact, for every $u \in \partial K_{p}$, from the definition of $A$ we have

$$
\begin{aligned}
\|A u\| & =\max \left|\iiint_{0}^{1} G_{1}(t, \delta) G_{2}(\delta, \tau) G_{3}(\tau, s) f(s, u(s)) d s d \tau d \delta\right| \\
& \leq C_{1} C_{2} C_{3} M_{1} M_{2}\left|\int_{0}^{1} G_{3}(s, s) f(s, u(s)) d s\right| \\
& \leq C_{1} C_{2} C_{3} M_{1} M_{2} \int_{0}^{1} G_{3}(s, s) \eta p d s \\
& =\|u\| .
\end{aligned}
$$

From (ii) of Lemma 2.8, we have

$$
i\left(A, K_{p}, K\right)=1 .
$$

Combining (3.14) and (3.19), we have

$$
\begin{gathered}
i\left(A, K_{R} \backslash \overline{K_{p}}, K\right)=i\left(A, K_{R}, K\right)-i\left(A, K_{p}, K\right)=-1, \\
i\left(A, K_{p} \backslash \overline{K_{r}}, K\right)=i\left(A, K_{p}, K\right)-i\left(A, K_{r}, K\right)=1 .
\end{gathered}
$$


Therefore, $A$ has fixed points $u_{1}$ and $u_{2}$ in $K_{p} \backslash \overline{K_{r}}$ and $K_{R} \backslash \overline{K_{p}}$, respectively, which means that $u_{1}(t)$ and $u_{2}(t)$ are positive solutions of $\operatorname{BVP}(1.6)-(1.7)$ and $0 \leq\left\|u_{1}\right\| \leq p \leq\left\|u_{2}\right\|$. The proof is completed.

Theorem 4.2. If $\bar{f}_{0}<L$ and $\bar{f}_{\infty}<L$ and (H4) is satisfied, then BVP(1.6)-(1.7) has at least two positive solutions: $u_{1}$ and $u_{2}$, such that $0 \leq\left\|u_{1}\right\| \leq p \leq\left\|u_{2}\right\|$.

Proof. According to the proof of Theorem 3.1, there exists $0<\omega<p<R_{2}<+\infty$, such that $0<r<\omega$ implies $i\left(A, K_{r}, K\right)=1$ and $R \geq R_{2}$ implies $i\left(A, K_{R}, K\right)=1$.

We now prove that $i\left(A, K_{p}, K\right)=0$ if $(H 4)$ is satisfied. In fact, for every $u \in \partial K_{p}$, from the proof of (i) of Theorem 3.1, we have

$$
\begin{aligned}
\left\|A u\left(\frac{1}{2}\right)\right\| & =\iiint_{0}^{1} G_{1}\left(\frac{1}{2}, \delta\right) G_{2}(\delta, \tau) G_{3}(\tau, s) f(s, u(s)) d s d \tau d \delta \\
& \geq \delta_{1} \delta_{2} \delta_{3} C_{12} C_{23} m_{1} \int_{1 / 4}^{3 / 4} G_{3}(s, s) v p d s \\
& =\|u\| .
\end{aligned}
$$

Therefore, $\|A u\| \geq\|A u(1 / 2)\| \geq\|u\|$, according to (i) of Lemma $2.8, i\left(A, K_{p}, K\right)=0$.

Combining (3.4) and (3.23), we have

$$
\begin{aligned}
& i\left(A, K_{R} \backslash \overline{K_{p}}, K\right)=i\left(A, K_{R}, K\right)-i\left(A, K_{p}, K\right)=1, \\
& i\left(A, K_{p} \backslash \overline{K_{r}}, K\right)=i\left(A, K_{p}, K\right)-i\left(A, K_{r}, K\right)=-1 .
\end{aligned}
$$

Therefore, $A$ has the fixed points $u_{1}$ and $u_{2}$ in $K_{p} \backslash \overline{K_{r}}$ and $K_{R} \backslash \overline{K_{p}}$, respectively, which means that $u_{1}(t)$ and $u_{2}(t)$ are positive solutions of $\operatorname{BVP}(1.6)-(1.7)$ and $0 \leq\left\|u_{1}\right\| \leq p \leq\left\|u_{2}\right\|$. The proof is completed.

Theorem 4.3. If $\underline{f}_{0}>\operatorname{L}$ and $\bar{f}_{\infty}<L$, and there exists $p_{2}>p_{1}>0$ that satisfies

(i) $f(t, u)<\eta p_{1}$ if $0 \leq t \leq 1$ and $0 \leq u \leq p_{1}$,

(ii) $f(t, u) \geq v p_{2}$ if $0 \leq t \leq 1$ and $\sigma p_{2} \leq u \leq p_{2}$,

then $B V P(1.6)-(1.7)$ has at least three positive solutions: $u_{1}, u_{2}$, and $u_{3}$, such that $0 \leq\left\|u_{1}\right\| \leq p_{1} \leq$ $\left\|u_{2}\right\| \leq p_{2} \leq\left\|u_{3}\right\|$.

Proof. According to the proof of Theorem 3.1, there exists $0<r_{0}<p_{1}<p_{2}<R_{3}<+\infty$, such that $0<r<r_{0}$ implies $i\left(A, K_{r}, K\right)=1$ and $R \geq R_{3}$ implies $i\left(A, K_{R}, K\right)=1$.

From the proof of Theorems 4.1 and 4.2, we have

$$
i\left(A, K_{p_{1}}, K\right)=1, \quad i\left(A, K_{p_{2}}, K\right)=0 .
$$


Combining the four afore-mentioned equations, we have

$$
\begin{gathered}
i\left(A, K_{R} \backslash \overline{K_{p_{2}}}, K\right)=i\left(A, K_{R}, K\right)-i\left(A, K_{p_{2}}, K\right)=1, \\
i\left(A, K_{p_{2}} \backslash \overline{K_{p_{1}}}, K\right)=i\left(A, K_{p_{2}}, K\right)-i\left(A, K_{p_{1}}, K\right)=-1 \\
i\left(A, K_{p_{1}} \backslash \overline{K_{r}}, K\right)=i\left(A, K_{p_{1}}, K\right)-i\left(A, K_{r}, K\right)=1 .
\end{gathered}
$$

Therefore, $A$ has the fixed points $u_{1}, u_{2}$, and $u_{3}$ in $K_{p_{1}} \backslash \overline{K_{r}}, K_{p_{2}} \backslash \overline{K_{p_{1}}}$, and $K_{R} \backslash \overline{K_{p_{2}}}$, which means that $u_{1}(t), u_{2}(t)$, and $u_{3}(t)$ are positive solutions of BVP(1.6)-(1.7) and $0 \leq\left\|u_{1}\right\| \leq p_{1} \leq$ $\left\|u_{2}\right\| \leq p_{2} \leq\left\|u_{3}\right\|$. The proof is completed.

\section{References}

[1] R. A. Gardner and C. K. R. T. Jones, “Traveling waves of a perturbed diffusion equation arising in a phase field model," Indiana University Mathematics Journal, vol. 39, no. 4, pp. 1197-1222, 1990.

[2] G. Caginalp and P. C. Fife, "Higher-order phase field models and detailed anisotropy," Physical Review. B, vol. 34, no. 7, pp. 4940-4943, 1986.

[3] T. Gyulov, G. Morosanu, and S. Tersian, "Existence for a semilinear sixth-order ODE," Journal of Mathematical Analysis and Applications, vol. 321, no. 1, pp. 86-98, 2006.

[4] L. A. Peletier and W. C. Troy, Spatial Patterns, vol. 45 of Progress in Nonlinear Differential Equations and their Applications, Birkhäuser Boston, Boston, Mass, USA, 2001.

[5] L. A. Peletier and V. Rottschäfer, "Large time behaviour of solutions of the Swift-Hohenberg equation," Comptes Rendus Mathématique. Académie des Sciences. Paris, vol. 336, no. 3, pp. 225-230, 2003.

[6] S. Tersian and J. Chaparova, "Periodic and homoclinic solutions of extended Fisher-Kolmogorov equations," Journal of Mathematical Analysis and Applications, vol. 260, no. 2, pp. 490-506, 2001.

[7] Y. Li, "Positive solutions of fourth-order boundary value problems with two parameters," Journal of Mathematical Analysis and Applications, vol. 281, no. 2, pp. 477-484, 2003.

[8] S. Fan, "The new root formula and criterion of cubic equation," Journal of Hainan Normal University, vol. 2, pp. 91-98, 1989 (Chinese).

[9] D. J. Guo and V. Lakshmikantham, Nonlinear Problems in Abstract Cones, vol. 5 of Notes and Reports in Mathematics in Science and Engineering, Academic Press, Boston, Mass, USA, 1988. 